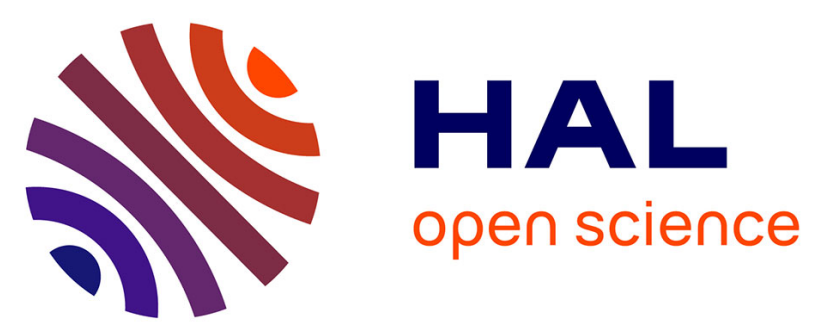

\title{
Group velocity coupling and synchronization-like phenomenon of two light beams based on coherent population oscillations
}

\author{
Sonia Melle, Oscar G Calderón, Marcelo Moreno
}

\section{- To cite this version:}

Sonia Melle, Oscar G Calderón, Marcelo Moreno. Group velocity coupling and synchronization-like phenomenon of two light beams based on coherent population oscillations. Journal of Physics B: Atomic, Molecular and Optical Physics, 2010, 43 (21), pp.215401. 10.1088/0953-4075/43/21/215401 . hal-00569862

\section{HAL Id: hal-00569862 https://hal.science/hal-00569862}

Submitted on 25 Feb 2011

HAL is a multi-disciplinary open access archive for the deposit and dissemination of scientific research documents, whether they are published or not. The documents may come from teaching and research institutions in France or abroad, or from public or private research centers.
L'archive ouverte pluridisciplinaire HAL, est destinée au dépôt et à la diffusion de documents scientifiques de niveau recherche, publiés ou non, émanant des établissements d'enseignement et de recherche français ou étrangers, des laboratoires publics ou privés. 


\title{
Group velocity coupling and synchronization-like phenomenon of two light beams based on coherent population oscillations
}

\author{
Sonia Melle, Oscar G. Calderón, Marcelo Moreno \\ Escuela Universitaria de Óptica, Universidad Complutense de Madrid, Arcos de \\ Jalón 118, 28037 Madrid, Spain \\ E-mail: smelle@fis.ucm.es
}

\begin{abstract}
We report on the control of the slow and fast light propagation velocity of two light beams (at $1550 \mathrm{~nm}$ and $980 \mathrm{~nm}$ ) through an erbium doped fiber amplifier by means of coherent population oscillations. We modulate the amplitude of both beams with the same frequency so that the relative phase between both modulations allows us to control their group velocities. The same group velocity, in absolute value, is achieved when beams are modulated in phase or phase opposition. The experimental results are explained by a rate equation model.
\end{abstract}




\section{Introduction}

Slow light has recently become a subject of intense research due to its potential applications for tunable delay lines which can be used for data synchronization [1]. Thus, much research has been focused on the control of the speed of light in optical fibers since these devices would be compatible with fiber-optic communication systems (see review about slow light in optical fibers [2]). Song et al. [3] and Okawachi et al. [4] demonstrated slow and fast light in optical fibers for the first time. The underlying mechanism used on those works, stimulated Brillouin scattering, consists in the interaction of two propagating waves, a pump wave and a Stokes wave, which generates an acoustic wave at the frequency difference of the pump and the Stokes fields. The slow light resonance can be placed at the desired wavelength by changing the frequency of the pump field. A related process, stimulated Raman scattering, has also been used in optical fibers to demonstrate an ultrafast all-optical controllable delay [5]. Electromagnetically induced transparency (EIT), a pioneering technique to produce slow light propagation, has been recently demonstrated experimentally in hollow-core photonic-bandgap fibers filled with acetylene [6]. This technique creates a very deep transparency window in an atomic absorption profile with almost null absorption. However, EIT medium should preserve quantum coherence, therefore dephasing processes limit this technique.

Coherent population oscillations (CPO) have been shown to be another physical mechanism which allows for the variation of group velocity in optical fibers at room temperature. The periodic modulation of the ground-state population at the beat frequency between a control and a probe field sharing a common atomic transition produces scattered light from the control field into the probe field leading to a decrease in the absorption of the probe field. This give rise to a narrow hole in the absorption profile for the probe field leading to slow light propagation [7]. The hole linewidth is proportional to the inverse of the relaxation lifetime of the excited level [8]. Note that this technique is not affected by dephasing processes, in contrast to EIT. However, the transparency depth achieved in CPO is smaller than the one obtained with EIT. Therefore, CPO could be affected by the residual absorption. By means of an additional pump to populate upper levels, the CPO induces a dip in the gain spectrum, leading to anomalous dispersion and fast light (group velocities greater than $c$ or even negative). A modification of group velocity by CPO in an erbium doped fiber (EDF) was reported for the first time by Schweinsberg et al [9], where an amplitudemodulated $1550 \mathrm{~nm}$ signal co-propagates with a $980 \mathrm{~nm}$ pump beam. They observed a change from sub- to superluminal propagation upon increasing pump power. The delay or advancement achieved saturates with both pump and signal powers, being this a limit in the delays and advancements obtained. Larger delays/advancements have been obtained for ultra-highly EDFs [10, 11, 12]. By using the same experimental system, fast light pulse propagation has been studied in more detail in [13, 14, 15]. Furthermore, a change from superluminal to subluminal propagation solely based upon increasing the beat frequency between the weak and the control field sharing a common atomic transition has been reported $[16,17]$. This peculiar behavior is due to the interplay between pump absorption and pump-power broadening of the spectral hole induced by CPO.

Recently, Arrieta-Yáñez et al have developed a novel mechanism for enhancing the fractional delay and advancement obtained in EDFs by one order of magnitude [18]. They force the population oscillations by modulating with the same frequency 
and simultaneously both, the $980 \mathrm{~nm}$ pump beam and the $1550 \mathrm{~nm}$ signal beam. The population oscillations are amplified by the pump modulation. Relative phase between both modulations was used as a knob for changing the propagation regime from ultraslow velocities to fast- or negative-velocities. The same physical mechanism was previously used by Stepanov et al [19] to control the phase shift of a modulated signal beam by modulating an additional beam simultaneously and with the same frequency. These two beams have different carrier frequencies, both within the fundamental absorption spectrum 1470-1570 $\mathrm{nm}_{\text {of }} \mathrm{Er}^{3+}$ ions.

Our aim in this work is to study the coupling of two light beams through the population oscillations and its effect in the propagation velocity of both beams. To this end, we induce a beat in both beams with the same frequency and analyze simultaneously the group velocity of both modulated beams. We use an EDF and the two light beams correspond to the usual signal (at $1550 \mathrm{~nm}$ ) and pump (at 980 $\mathrm{nm}$ ) beams. This method allows us to control the group velocity of both beams by means of the relative phase between both beats. We analyze under which conditions the coupling between the two beams makes that both propagate with the same group velocity, that is, the conditions that lead to a group velocity synchronization-like phenomenon.

The paper is organized as follows. The theoretical model used to study the propagation of two amplitude periodically-modulated beams through an EDF using CPO is described in section 2. The experimental setup is described in section 3 . The experimental results and simulations are presented in section 4 . The final conclusions are summarized in section 5 .

\section{Propagation equations}

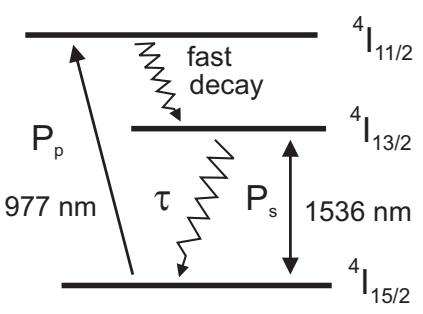

Figure 1. Three-level system for $\mathrm{Er}^{3+}$ ions. Wavelengths for the signal and pump beams.

The propagation of an amplitude periodically-modulated signal through an EDF has been modeled in previous works on CPO by using both semiclassical and rate equation procedures [7, 9]. We consider the erbium ions as three-level atomic systems interacting with a power-modulated $1536 \mathrm{~nm}$ signal which couples the transition ${ }^{4} I_{15 / 2} \longrightarrow{ }^{4} I_{13 / 2}$ and a $977 \mathrm{~nm}$ pump laser which pump the ions onto the upper state ${ }^{4} I_{11 / 2}$ (see figure 1). We also assume a fast decay from the upper state ${ }^{4} I_{11 / 2}$ to the metastable state $\left({ }^{4} I_{13 / 2}\right)$. We obtain the rate equation for $N_{1}$ (the population of the ground state normalized to the total ion density $\rho$ ):

$$
\frac{\partial N_{1}}{\partial t}=\frac{1}{\tau}\left[1-N_{1}+\frac{\beta_{s}}{1+\beta_{s}} P_{s}\left(1-N_{1}\right)-\frac{1}{1+\beta_{s}} P_{s} N_{1}-P_{p} N_{1}\right]
$$


where the four terms on the right hand side of the equation stand for the spontaneous emission from the metastable state, the stimulated emission from the metastable state, the absorption to the metastable state, and the absorption to the upper level, respectively. Here $t$ is the time variable. $P_{s}$ is the signal power normalized to the signal saturation power $P_{s}^{s a t} \equiv \hbar \omega_{s} A_{s} /\left(\tau\left(\sigma_{21}+\sigma_{12}\right)\right)$, where $\tau$ is the lifetime of the metastable state, $\sigma_{21}$ is the emission cross section at the signal frequency $\omega_{s}, \sigma_{12}$ is the absorption cross section at the signal frequency $\omega_{s}$, and $A_{s}$ is the signal mode area. The ratio between the signal cross sections is $\beta_{s} \equiv \sigma_{21} / \sigma_{12} . P_{p}$ is the pump power normalized to the pump saturation power $P_{p}^{s a t} \equiv \hbar \omega_{p} A_{p} /\left(\tau \sigma_{13}\right)$, where $\sigma_{13}$ is the absorption cross section at the pump frequency $\omega_{p}$, and $A_{p}$ is the pump mode area. $\eta_{s} \equiv A_{c} / A_{s}$ is the ratio between the fiber core area and the signal mode area, and $\eta_{p} \equiv A_{c} / A_{p}$ is the ratio between the fiber core area and the pump mode area.

The behavior of the signal and pump powers along the fiber can be described by the following propagation equations:

$$
\begin{aligned}
& \frac{\partial P_{s}}{\partial z}+\frac{n_{g s}}{c} \frac{\partial P_{s}}{\partial t}=\sigma_{12} \rho\left[\beta_{s}-\left(1+\beta_{s}\right) N_{1}\right] \eta_{s} P_{s} \\
& \frac{\partial P_{p}}{\partial z}+\frac{n_{g p}}{c} \frac{\partial P_{p}}{\partial t}=-\sigma_{13} \rho N_{1} \eta_{p} P_{p}
\end{aligned}
$$

where $z$ is the distance along the fiber. Here $n_{g s}$ and $n_{g p}$ are the group indexes of the signal and pump in the fiber glass, respectively.

The signal is modulated as follows: $P_{s}=P_{s 0}+P_{s m} \cos \left(2 \pi f_{m} t\right)$, where $P_{s 0}$ is the average signal power, $P_{s m}$ is the modulation amplitude (modulation depth $\left.P_{s m} / P_{s 0}\right)$, and $f_{m}$ is the modulation frequency. Due to this modulation, a periodic coherent oscillation of the population of the ground state occurs at this frequency $\left(N_{1}=N_{1 s t}+N_{1 c} \cos \left(2 \pi f_{m} t\right)+N_{1 s} \sin \left(2 \pi f_{m} t\right)\right)$ which produces a hole in the absorption spectrum for the modulated part of the signal. Thus, a slow light propagation of $P_{s m}$ takes place. The presence of the pump beam allows to turn the delay into advancement (fast light), depending on the value of the pump power. However, the magnitude of the advancement obtained remains nearly constant when certain pump level has been achieved [7, 9, 10, 16, 20].

Here, we also modulate the pump beam with the same frequency as the signal beam, being the pump power made up of an average pump power $P_{p 0}$, and a sinusoidal part with amplitude $P_{p m}$ varying at frequency $f_{m}$, i.e., $P_{p}=P_{p 0}+P_{p m} \cos \left(2 \pi f_{m} t-\varphi\right)$. Note that the pump beam could be out of phase with the signal beam by a magnitude $\varphi$. Thus, the stationary part $\left(N_{1 s t}\right)$ and the oscillating parts $\left(N_{1 c}\right.$ and $\left.N_{1 s}\right)$ of the population of the ground state will be given by:

$$
\begin{aligned}
& N_{1 s t}=\frac{1}{w_{c}}\left(1+\frac{\beta_{s}}{1+\beta_{s}} P_{s 0}\right), \\
& N_{1 c}=\frac{w_{c} P_{s m}}{w_{c}^{2}+w_{m}^{2}}\left(\frac{\beta_{s}}{1+\beta_{s}}-N_{1 s t}\right)-\frac{N_{1 s t} P_{p m}}{w_{c}^{2}+w_{m}^{2}}\left(w_{c} \cos \varphi-w_{m} \sin \varphi\right), \\
& N_{1 s}=\frac{w_{m} P_{s m}}{w_{c}^{2}+w_{m}^{2}}\left(\frac{\beta_{s}}{1+\beta_{s}}-N_{1 s t}\right)-\frac{N_{1 s t} P_{p m}}{w_{c}^{2}+w_{m}^{2}}\left(w_{m} \cos \varphi+w_{c} \sin \varphi\right),
\end{aligned}
$$

where $w_{m} \equiv 2 \pi f_{m} \tau$ is the dimensionless modulation frequency and $w_{c} \equiv 1+P_{s 0}+P_{p 0}$ is a dimensionless frequency which determines the width of the transparency hole created in the absorption or gain spectrum by means of CPO. This magnitude roughly 
measures the maximum modulation bandwidth that can experience the full slow or fast light effect [21] and it can be increased with the signal and pump powers. The expression obtained for the out of phase part of the population oscillation $N_{1 s}$ presents two terms: the first one, which is proportional to $P_{s m}$, is responsible of the delay/advancement experienced by the modulated part of the signal. The second term, which is proportional to $P_{p m}$, arises from the modulation imposed to the pump beam and incorporates explicitly the phase delay $\varphi$. We will show that by adjusting the value of $\varphi$, the delay and advancement experienced by the modulated part of the signal can be controlled.

Finally, we can obtain the propagation equations for the average powers and the amplitudes of the modulation of the signal and pump beams, and for the phase shift experienced by the periodic part of the signal and pump beams due to CPO:

$$
\begin{aligned}
\frac{d P_{s 0}}{d z^{\prime}} & =\alpha_{s} P_{s 0}\left[\beta_{s}-\left(1+\beta_{s}\right) N_{1 s t}\right] \\
\frac{d P_{p 0}}{d z^{\prime}} & =-\alpha_{p} P_{p 0} N_{1 s t} \\
\frac{d P_{s m}}{d z^{\prime}} & =-\alpha_{s}\left(1+\beta_{s}\right) P_{s 0} N_{1 c}+\alpha_{s}\left[\beta_{s}-\left(1+\beta_{s}\right) N_{1 s t}\right] P_{s m}, \\
\frac{d P_{p m}}{d z^{\prime}} & =-\alpha_{p} P_{p 0}\left(N_{1 c} \cos \varphi+N_{1 s} \sin \varphi\right)-\alpha_{p} P_{p m} N_{1 s t} \\
\frac{d \phi_{s}}{d z^{\prime}} & =\alpha_{s}\left(1+\beta_{s}\right) \frac{P_{s 0}}{P_{s m}} N_{1 s} \\
\frac{d \phi_{p}}{d z^{\prime}} & =\alpha_{p} \frac{P_{p 0}}{P_{p m}}\left(N_{1 s} \cos \varphi-N_{1 c} \sin \varphi\right)
\end{aligned}
$$

where the distance has been normalized to the fiber length $L$ such that $z^{\prime} \equiv z / L$. $\alpha_{s} \equiv L \sigma_{12} \rho \eta_{s}$ is the dimensionless signal absorption coefficient and $\alpha_{p} \equiv L \sigma_{13} \rho \eta_{p}$ is the dimensionless pump absorption coefficient. We neglect the term with the temporal derivative in equations (2) and (3). This term leads to a small delay (delay due to the fiber glass refractive index dispersion) compared to the delay due to CPO. The fractional delay of the signal (time delay normalized to the time duration of the signal) at the end of the fiber can be obtained from the signal phase shift at $z=L$, i.e., $F_{s} \equiv \phi_{s}(z=L) /(2 \pi)$. While for the pump it will be $F_{p} \equiv \phi_{p}(z=L) /(2 \pi)$.

\section{Experimental setup}

The experimental setup is depicted in figure 2 and consists of an EDF in the forwardpumped configuration. The signal beam comes from a pigtailed distributed feedback laser diode operating at $1536 \mathrm{~nm}$ provided with a current and temperature controller which allows us to operate the laser at room temperature. This laser beam couples the transition between the erbium ground state ${ }^{4} I_{15 / 2}$ and the metastable state ${ }^{4} I_{13 / 2}$ (see figure 1). The signal beam is split into two beams: one part of the beam (1\%) is sent directly to a switchable-gain amplified InGaAs photodetector, which is used as reference. The other part of the beam (99\%) goes through the EDF under study and then to an identical photodetector. The EDF is pumped by a co-propagating beam coming from a pigtailed laser diode operating at $977 \mathrm{~nm}$ provided also with a current and temperature controller. This laser beam couples the erbium ground state with the fast decay upper pump level $\left({ }^{4} I_{11 / 2}\right)$ (see figure 1 ). The pump beam is also split 


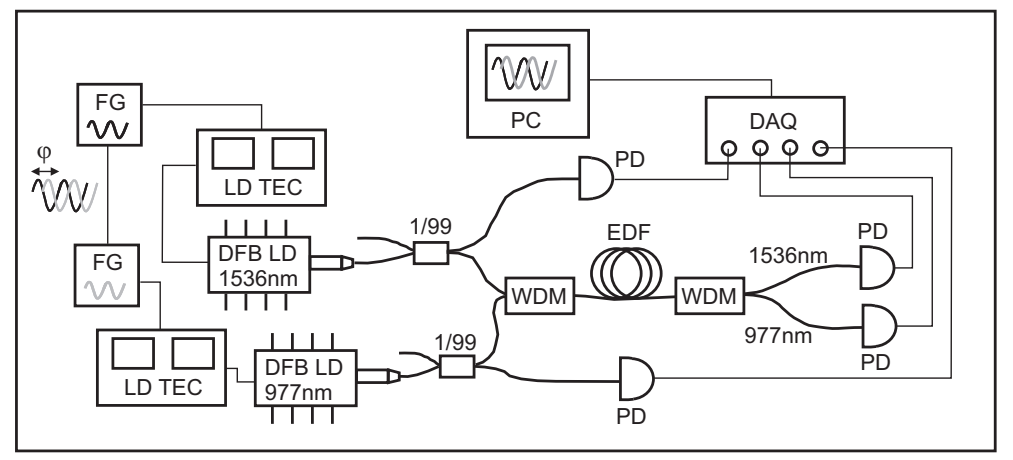

Figure 2. Experimental setup scheme. LD TEC: laser diode and temperature controller; FG: function generator; $\varphi$ : relative phase between the pump modulation and the signal modulation; DFB LD: distributed feedback laser diode; WDM: wavelength division multiplexer; EDF: erbium doped fiber; PD: photodetector; DAQ: data acquisition card; PC: personal computer.

into two beams: one part of the beam (1\%) is sent directly to a photodetector, which is used as reference. The other part of the beam (99\%) goes through the EDF and then to an identical photodetector. The signal and pump beams are injected into the EDF through a wavelength division multiplexer. The pump and the signal beams at the output of the EDF are separated by means of an identical wavelength division multiplexer. Both laser powers are sinusoidally modulated with the same modulation frequency $f_{m}$ by using two synchronized function generators which allow us to control the relative phase $\varphi$ between both laser powers at the input of the fiber. Four signals (the reference signal, the signal propagated through the EDF, the reference pump, and the pump propagated through the EDF) are recorded with a fast data acquisition card and transferred to a computer for analysis. The experiment is controlled with a LabView program. We compute the time delay or advancement of the signal $t_{d s}$ from the correlation of the reference signal and the signal propagated through the EDF. Similarly, we compute the time delay or advancement of the pump $t_{d p}$ from the correlation of the reference pump and the pump propagated through the EDF. Then, the fractional delay/advancement of the signal is defined as $F_{s} \equiv t_{d s} f_{m}$, while the fractional delay/advancement of the pump is defined as $F_{p} \equiv t_{d p} f_{m}$. We used a 0.1 m-long single mode $\mathrm{Al}_{2} \mathrm{SiO}_{5}$-glass-based fiber highly doped with $\mathrm{Er}^{3+}$ ions (ion density $\rho=6.3 \times 10^{25} \mathrm{~m}^{-3}$ ).

\section{Experimental results and simulations}

We have carried out two different set of experiments. In the first one, we analyze the enhancement of the slow and fast light propagation effects produced when forcing the population oscillations with a modulated pump. In the second one, we study the coupling of both modulated beams.

In order to theoretically reproduce the experimental results, we numerically solve the propagation equations (7)-(12). We have considered the following parameter values which are consistent with values previously used in other works [9, 22]: the signal cross section is $\sigma_{12}=\sigma_{21}=5 \times 10^{-25} \mathrm{~m}^{2}$, the pump cross section is $\sigma_{13}=3 \times 10^{-25} \mathrm{~m}^{2}$, the signal mode area is $A_{s} \simeq 33 \mu \mathrm{m}^{2}$ (signal mode field diameter $6.5 \mu \mathrm{m}$ ), the pump mode 
area is $A_{p} \simeq 20 \mu \mathrm{m}^{2}$ (pump mode field diameter $5.0 \mu \mathrm{m}$ ), the fiber core area is $A_{c} \simeq 14$ $\mu \mathrm{m}^{2}$ (fiber core diameter $4.2 \mu \mathrm{m}$ ), the lifetime of the metastable state is $\tau \simeq 9 \mathrm{~ms}$. Using these parameters, the signal saturation power is $P_{s}^{s a t} \simeq 0.5 \mathrm{~mW}$ and the pump saturation power is $P_{p}^{s a t} \simeq 1.5 \mathrm{~mW}$. The dimensionless signal absorption coefficient is $\alpha_{s} \simeq 1.35$, and the dimensionless pump absorption coefficient is $\alpha_{p} \simeq 1.33$. We used the insertion losses in the EDF as fitting parameters to reproduce the experimental curves. Thus, we considered an insertion losses of 0.8 for the signal beam and 0.65 for the pump beam. We are not considering changes in the refractive index due to nonlinear effects such as Kerr type nonlinearity since we are not working in a high power regimen.

\subsection{Signal delay controlled by the modulated pump}

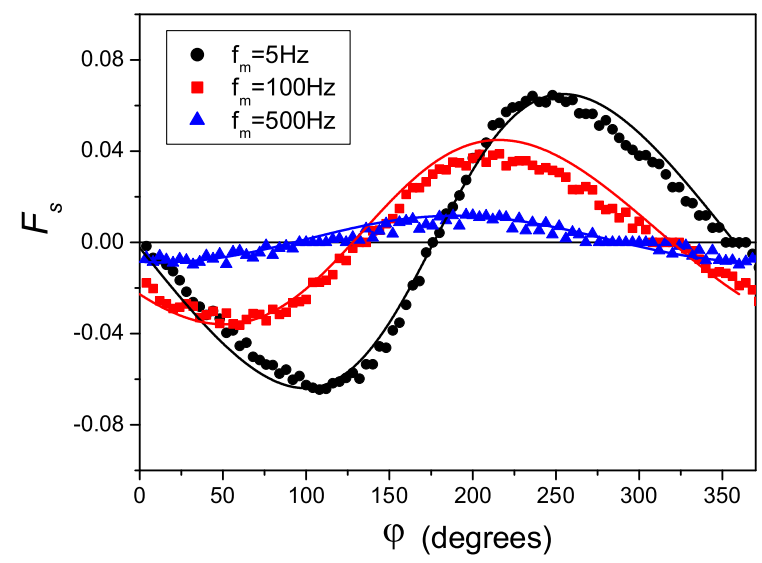

Figure 3. Fractional advancement of the signal as a function of the relative phase between the signal and pump modulated beams for three different modulation frequencies. Experiments (symbols) and simulations (lines). Signal power $=0.14$ $\mathrm{mW}\left(P_{s 0}=0.24\right)$. Pump power $=10 \mathrm{~mW}\left(P_{p 0}=4.3\right)$. Modulation depths $P_{s m} / P_{s 0}=P_{p m} / P_{p 0} \simeq 0.5$.

First of all, we have analyzed the viability of controlling the propagation regime of the signal by means of the pump modulation. In figure 3 we show the dependence of the signal fractional delay $F_{s}$ with the relative phase between the signal and pump modulated beams $\varphi$. We modulate the signal power $(0.14 \mathrm{~mW})$ and the pump power $(10 \mathrm{~mW})$ with the same modulation depth $\left(P_{s m} / P_{s 0}=P_{p m} / P_{p 0} \simeq 0.5\right)$ and frequency. Different curves correspond to different modulation frequencies. The experimental results (symbols) have been plotted together with the simulated curves (lines) obtained by numerically solving the system of equations (7)-(12). We obtain ultra slow group velocities or very fast group velocities (even negatives) depending on the relative phase $\varphi$. It can be seen that the value of $\varphi$ that leads to the maximum fractional delay or advancement shifts with the modulation frequency. For low frequencies, the maximum delay takes place around $\pi / 2$ while the maximum advancement takes place around $3 \pi / 2$. As the modulation frequency increases, the position of the maximum delay shifts and tends to 0 while the position of the maximum advancement tends 
to $\pi$. This behavior can be analytically explained by neglecting the attenuation or amplification of the signal and pump powers (undepleted solution) in equation (11). In this case, the phase shift of the signal is obtained by assuming implicitly the absence of $z$-dependence in the problem:

$\phi_{s}(z=L) \simeq \frac{\alpha_{s} P_{s 0}}{\left(w_{c}^{2}+w_{m}^{2}\right) w_{c}}\left[w_{m}\left(\beta_{s} P_{p 0}-1\right)-\frac{P_{p m}}{P_{s m}}\left(1+\beta_{s}+\beta_{s} P_{s 0}\right) \sin \left[\varphi+\xi_{m}\right]\right]$

where $\xi_{m} \equiv \arctan \left(w_{m} / w_{c}\right)$. By analyzing equation (13) we obtain that the maximum fractional advancement/delay takes place at $\varphi=(\pi / 2)+n \pi-\xi_{m}$ (with $\left.n=0,1\right)$. Then, at low modulation frequencies $\left(w_{m}<w_{c}\right), \xi_{m} \simeq 0$, so the minimum and the maximum take place around $\pi / 2$ and $3 \pi / 2$, respectively; whereas at high modulation frequencies $\left(w_{m}>w_{c}\right), \xi_{m} \simeq \pi / 2$, so the minimum and the maximum take place around 0 and $\pi$, respectively, in agreement with the experimental results.

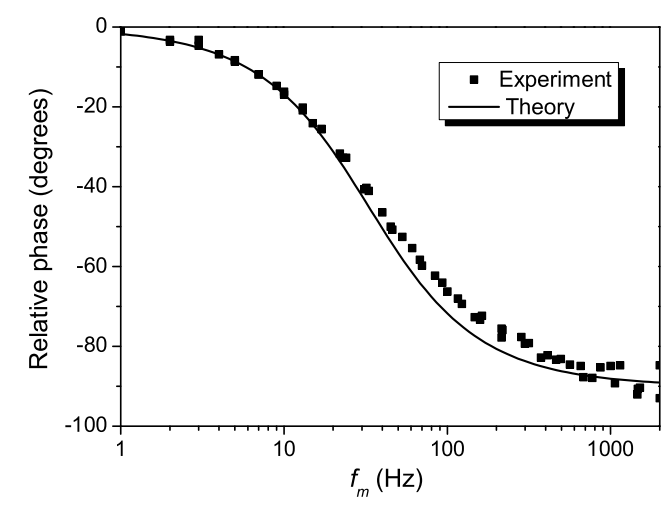

Figure 4. Phase between the signal and the pump beams as a function of the modulation frequency. Experiment (symbols) and theory (line). Signal power = $0.1 \mathrm{~mW}\left(P_{s 0}=0.17\right)$. Pump power $=1.5 \mathrm{~mW}\left(P_{p 0}=0.66\right)$. Pump modulation depth $P_{p m} / P_{p 0}=0.5$.

With the aim of better understanding the above results, we are going to take into account that the pump beam dominates the population oscillation since the pump power is much higher than the signal power, so its modulation amplitude is higher too. Thus, when only the pump beam is modulated, an oscillation will be induced in the signal beam due to the population oscillations. It is easy to analytically show that, in this particular case, the signal beam will oscillate with a phase shift of $\xi_{m}$ with respect to the pump beam. In fact, this phase shift is the phase difference of the population oscillations with respect to the oscillation of the pump beam. In other words, the signal beam will oscillate in phase with the population oscillations which are induced by the modulated pump. In order to experimentally check this result, we have measured the phase shift between the signal and the pump beams when modulating a $1.5 \mathrm{~mW}$ pump beam with a constant signal beam of $0.1 \mathrm{~mW}$. In figure 4 we plot the relative phase between the signal and pump beams as a function of the modulation frequency. We observe that for low modulation frequencies, the signal oscillates in phase with the pump beam, and for high modulation frequencies, the shift between 
the pump beam and the signal beam is $\pi / 2$. As expected, the relative phase between the signal and pump beams behaves as $\xi_{m}$, as can be seen in figure 4 (solid line).

So the oscillation that the pump induces in the signal has a phase shift of $\xi_{m}$ with respect to the pump oscillation. When we modulate both beams simultaneously, as in figure 3 , the signal oscillation will not affect to the population oscillations since the signal power and, thus, the signal modulation amplitude are smaller than the corresponding to the pump. Then, at low modulation frequencies $\left(\xi_{m} \simeq 0\right)$, i.e., when the oscillations of the pump and the population are in phase, the maximum delay/advancement takes place when signal and pump are initially out of phase. However, at high modulation frequencies $\left(\xi_{m} \simeq \pi / 2\right)$, i.e., when the oscillations of the pump and the population are dephased by $\pi / 2$, the signal will have a maximum delay or advancement when the signal oscillations are initially in phase with the pump oscillations. Thus, the maximum fractional delay (or advancement) occurs when the phase difference between the population and the signal beam is $\pi / 2$ (or $3 \pi / 2$ ) for all modulation frequencies, i.e., $\varphi+\xi_{m}=\pi / 2$ (or $3 \pi / 2$ ).

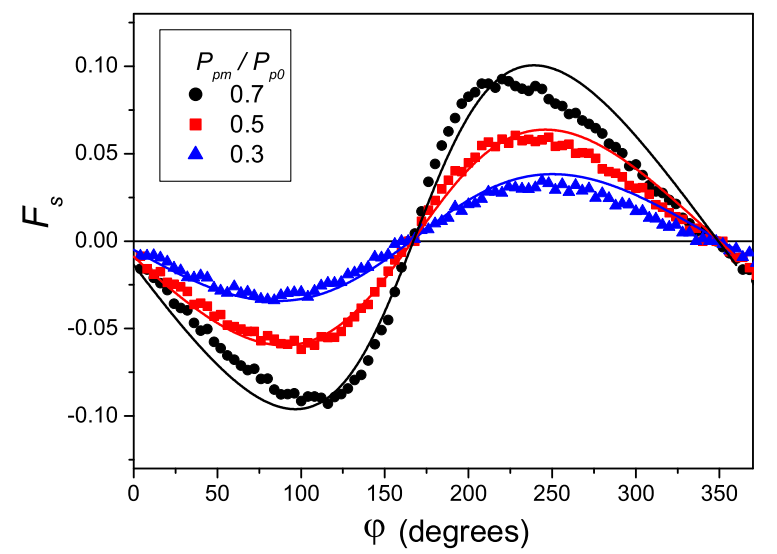

Figure 5. Fractional advancement of the signal as a function of the relative phase between the signal and pump modulated beams for three different pump modulation depths. Experiments (symbols) and simulations (lines). $f_{m}=20 \mathrm{~Hz}$. The rest of parameters are the same than the ones used in figure 3.

We have also analyzed the behavior of $F_{s}$ with the amplitude of the pump modulation $P_{p m}$. Figure 5 shows the experimental (symbols) and the simulated (lines) fractional advancement of the signal $F_{s}$ versus $\varphi$ when we modulate the pump and the signal powers at a low modulation frequency of $f_{m}=20 \mathrm{~Hz}$. The simulated curves have been obtained by numerically solving the system of equations (7)-(12). Different curves correspond to different modulation depths of the pump beam. The rest of parameters are the same than the ones used in figure 3. We see how the maximum advancement and maximum delay increase linearly with the modulation amplitude, in agreement with the analytical expression (see equation (13)). Note that, for the largest pump modulation depth, the shape of the curve developed by $F_{s}(\varphi)$ slightly deviates from a sinusoidal profile (see circles in figure 5), i.e., from the prediction given by equation (13). This analytical expression was obtained by assuming implicitly the absence of $z$-dependence in the problem. However, the phase shift of the signal is 
coupled to the amplitude of the signal modulation, in contrast to what occurs without pump modulation. Therefore, even in the undepleted case, for large amplitudes of the pump modulation we must take into account the spatial variation of the amplitude of the modulated part of the signal in order to accurately explain the behavior of the phase shift of the signal. In order to show this effect, let us consider that the spatial variation of the amplitude of the signal modulation $P_{s m}$ is very small so that:

$$
P_{s m}(z) \simeq P_{s m}(0)+\frac{z \alpha_{s}}{L} P_{p m} \cos \varphi \frac{P_{s 0}\left(1+\beta_{s}+\beta_{s} P_{s 0}\right)}{w_{c}^{2}},
$$

where we have considered a low modulation frequency, i.e., $w_{m}<w_{c}$. By using this expression we calculate the phase shift $\phi_{s}$ at the fiber output:

$$
\phi_{s}(z=L) \propto-\sin \varphi\left[1-P_{p m} \cos \varphi \frac{\alpha_{s} P_{s 0}\left(1+\beta_{s}+\beta_{s} P_{s 0}\right)}{2 w_{c}^{2} P_{s m}(0)}\right],
$$

which shows a deviation from the sinusoidal profile. This correction leads to a shift of the position of the maximum delay towards larger values of $\varphi$ whereas leads to a shift of the position of the maximum advancement in the opposite direction. The magnitude of this shift increases as the amplitude of the pump modulation increases, in agreement with the experimental results (see figure 5). This deviation of the $F_{s}(\varphi)$ curve from the sinusoidal profile is due to the coupling of the phase shift $\phi_{s}$ with the amplitude of signal modulation $P_{s m}$, which in fact, arises when modulating the pump.

Finally, we compare the magnitude of the signal fractional delay/advancement obtained in the presence or absence of modulation of the pump beam. To this aim, we have measured several curves of $F_{s}$ versus $f_{m}$ in the absence and in the presence of modulation of the pump beam. In the presence of modulation, we have also varied the values of the relative phase between the signal and pump modulated beams $\varphi$. These results are plotted in figure 6 . When the pump is not modulated, the maximum fractional delay or advancement that can be achieved within the undepleted approximation is given by $\pm \alpha_{s} /(16 \pi)$ which, for our experimental system, is close to \pm 0.025 . Note that when this approximation is not valid (high optical densities) a more complex relation of the maximum fractional delay with the fiber length is obtained [17]. These two optimum cases have been measured when we modulate the signal with an amplitude of $P_{s m}=0.3 P_{s 0}$. Optimum delay is achieved without pump, at the optimum modulation frequency $w_{m} \simeq w_{c}$, and an average signal power of $0.5 \mathrm{~mW}$ close to the signal saturation power $P_{s 0} \simeq 1$ (see squares in figure $6(\mathrm{a})$ ). Maximum advancement is achieved increasing the signal power to $2 \mathrm{~mW}$ and applying a high pump power of $23 \mathrm{~mW}$ (see circles in figure $6(\mathrm{a})$ ). In order to analyze the effect of the periodic modulation of the pump beam, we consider similar parameters to the ones used without pump modulation while we periodically modulate the pump power such that $P_{p m}=0.7 P_{p 0}$. Different curves shown in figure $6(\mathrm{~b})-(\mathrm{d})$ correspond to different values of the relative phase between the signal and pump modulated beams $\varphi$. The simulated curves have also been plotted in figure 6 (lines). We observe that the delay and advancement obtained when modulating the pump beam is around one order of magnitude larger than the one obtained without pump modulation. Furthermore, the behavior exhibited by the signal delay strongly depends on $\varphi$. We observe that for values of $\varphi$ around $\pi / 2$, delay is achieved for all values of $f_{m}$ (see figure $6(\mathrm{~b})$ ), whereas for values of $\varphi$ around $3 \pi / 2$, advancement is achieved for all modulation frequencies (see figure $6(\mathrm{~d})$ ). Note that for intermediate values of $\varphi$, around $\pi$, an increase in the 

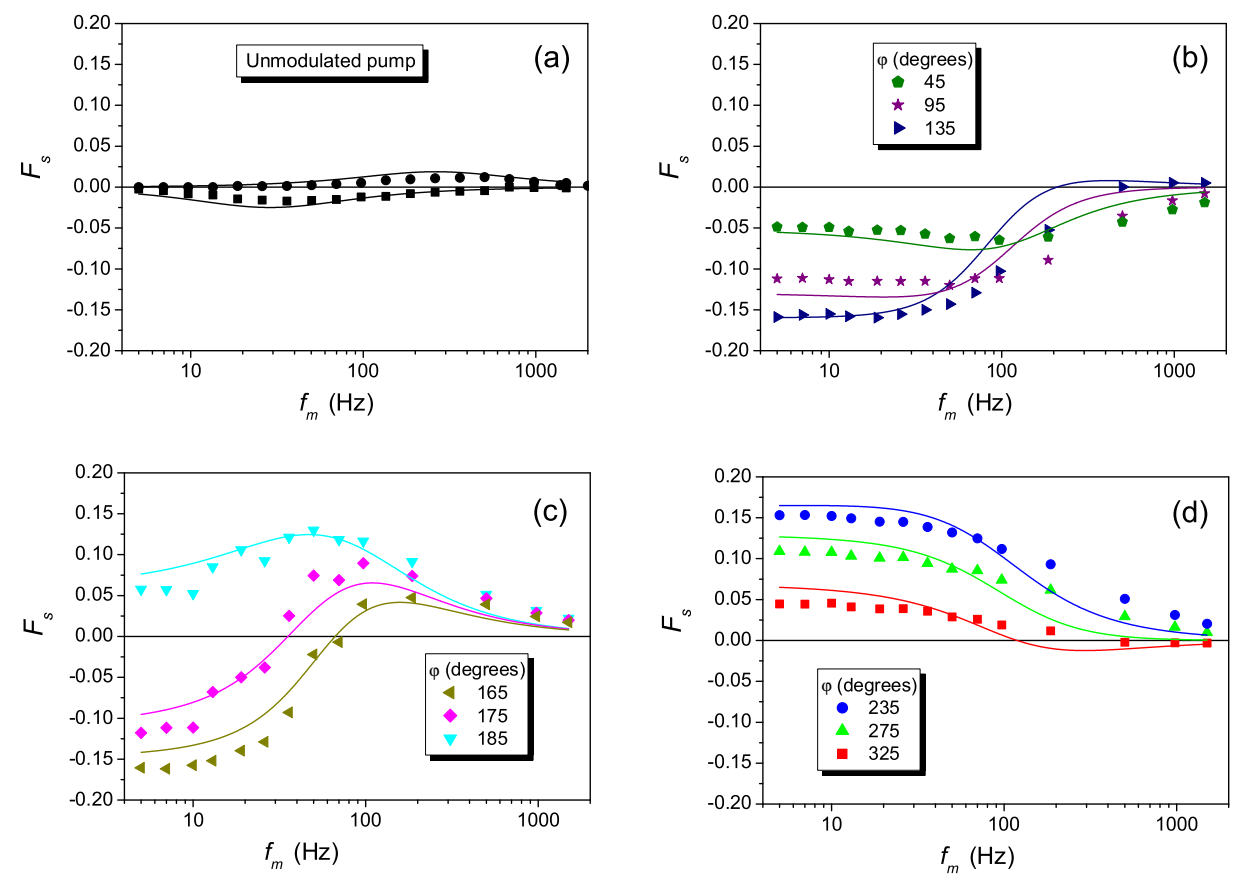

Figure 6. Fractional advancement of the signal as a function of the modulation frequency: (a) for the unmodulated pump case; and (b)-(d) when modulating the pump beam and for different relative phases between the signal and pump modulated beams $\varphi$. Experiments (symbols) and simulations (lines). (a) Optimum delay (squares): Signal power $=0.5 \mathrm{~mW}\left(P_{s 0}=0.9\right)$ with $P_{s m}=$ $0.3 P_{s 0}$, and pump power $P_{p 0}=0 \mathrm{~mW}$; Optimum advancement (circles): Signal power $=2 \mathrm{~mW}\left(P_{s 0}=3.4\right)$ with $P_{s m}=0.3 P_{s 0}$, and pump power $=23 \mathrm{~mW}\left(P_{p 0}\right.$ $=10)$. (b)-(d) Signal power $=0.5 \mathrm{~mW}\left(P_{s 0}=0.9\right)$ with $P_{s m}=0.4 P_{s 0}$, and pump power $=10 \mathrm{~mW}\left(P_{p 0}=4.4\right)$ with $P_{p m}=0.7 P_{p 0}$.

modulation frequency switches from delay to advancement (see figure 6 (c)). These results have been reproduced by the simulations.

The signal fractional advancement can be related with the refractive index of the material at the probe field frequency which usually shows a dispersive-like profile. However, curves in figure 6 show a dispersive- or absorptive-like shape depending on the value of the relative phase between the signal and pump modulated beams. In order to clarify this behavior, we analyze theoretically the evolution of the fractional advancement and the modulation gain (the ratio of the output to input modulation depths) for the signal and the pump as a function of the modulation frequency. In figure 7 we plot that dependencies for four representative values of the relative phase between the signal and pump modulated beams and for the same parameters used in figure 6. As we said before, when the pump modulation amplitude is much higher than the signal modulation amplitude, the population oscillations are mainly due to 

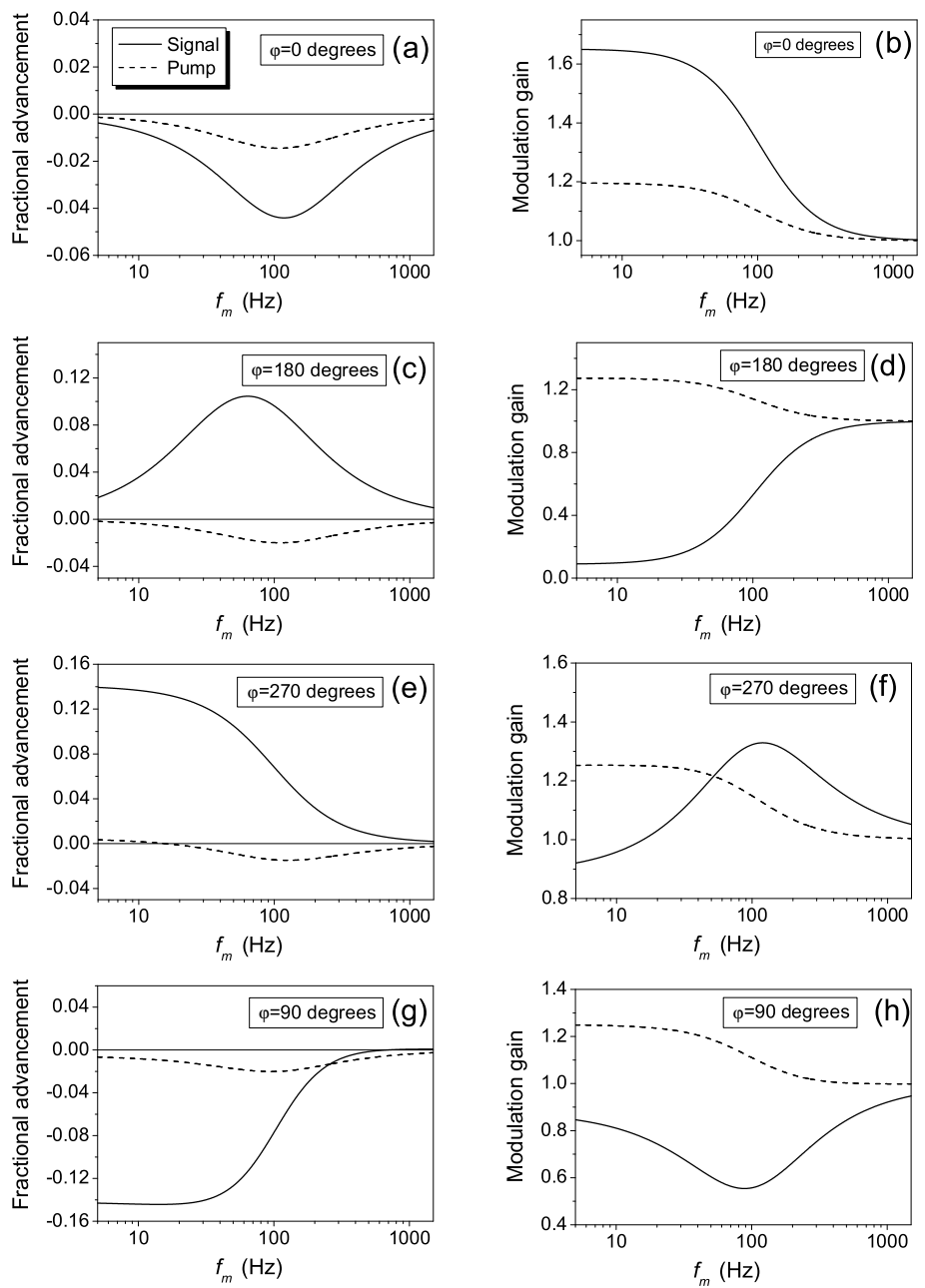

Figure 7. Simulated fractional advancement of the signal (solid line) and pump (dashed line) as a function of the modulation frequency for different relative phases between the signal and pump modulated beams: $\varphi=0$ (a) $\varphi=\pi$ (c), $\varphi=3 \pi / 2(\mathrm{e})$, and $\varphi=\pi / 2(\mathrm{~g})$. Simulated modulation gain for the signal $\left[\left(P_{s m} / P_{s 0}\right)_{\text {out }} /\left(P_{s m} / P_{s 0}\right)_{i n}\right]$ (solid line) and pump (dashed line) as a function of the modulation frequency for $\varphi=0$ (b) $\varphi=\pi$ (d), $\varphi=3 \pi / 2$ (f), and $\varphi=\pi / 2$ (h). $P_{s 0}=0.9$ with $P_{s m}=0.4 P_{s 0}$, and $P_{p 0}=4.4$ with $P_{p m}=0.7 P_{p 0}$.

the pump beam. In that case, the dependence of the pump phase shift with the modulation frequency is given by $\phi_{p} \propto-\omega_{m} /\left(\omega_{c}^{2}+\omega_{m}^{2}\right)$ while the dependence of the pump modulation amplitude with $\omega_{m}$ is $P_{p m} \propto \omega_{c} /\left(\omega_{c}^{2}+\omega_{m}^{2}\right)$. That is, $\phi_{p}$ shows the form of a dispersive-like curve while $P_{p m}$ follows the form of an absorptive-like curve which shows the hole produced by the population oscillations in the pump absorption spectrum. 
Both, the dispersive and absorptive phenomena that characterize $\phi_{p}$ and $P_{p m}$, contribute to the phase shift and also to the modulation amplitude of the signal beam through the relative phase $\varphi$. Thus, for $\varphi=0$ or $\varphi=\pi, \phi_{s}$ follows a dispersive-like curve whereas $P_{s m}$ develops an absorptive-like curve similarly to the behavior of $\phi_{p}$ and $P_{p m}$, respectively. In particular, for $\varphi=0, \phi_{s}$ and $P_{s m}$ behaves in the same way than $\phi_{p}$ and $P_{p m}$, respectively, as can be seen in figures 7 (a) and (b). As the signal modulation gain measures the ratio of the transmittance of the modulated part of the signal and the average signal power, we see in figure 7 (b) that the modulated part of the signal $P_{s m}$ exhibits more gain than the average signal power $P_{s 0}$. Thus, the signal delay can be explained as an antihole in the gain spectrum (gain increase) for $P_{s m}$ generated by the population oscillations governed by the pump. For $\varphi=\pi, \phi_{s}$ and $P_{s m}$ behaves in a similar manner although with opposite sign (see figures 7 (c) and (d)). In this case, the signal advancement is due to the hole produced in the gain spectrum by the population oscillations. More complex behaviors are obtained for $\varphi=\pi / 2$ or $\varphi=3 \pi / 2$. In those particular cases, there is an interchange between the roles of $\phi_{s}$ and $P_{s m}$. That is, $\phi_{s}$ develops an absorptive-like curve following $P_{p m}$ whereas $P_{s m}$ develops a dispersive-like curve following $\phi_{p}$. The case of $\varphi=3 \pi / 2$ is shown in figures 7 (e) and (f). We see that $\phi_{s}$ resembles the spectral hole obtained for the pump whereas $P_{s m}$ looks like the dispersive-like curve developed for the pump phase shift. In this case, the modulated part of the signal $P_{s m}$ exhibits more gain than the average signal power $P_{s 0}$ and this phenomenon occurs for frequencies around $\omega_{c}$ (see figures 7 (e) and (f)). This shifted gain peak could be seen as a hole in the gain spectrum around $\omega_{m}=0$, leading to signal advancement. For $\varphi=\pi / 2$, a similar result is obtained (see figures $7(\mathrm{~g})$ and $(\mathrm{h})$ ). In this case, the modulated part of the signal $P_{s m}$ exhibits less gain than the average signal power $P_{s 0}$ and this phenomenon occurs again for frequencies around $\omega_{c}$ (see figures $7(\mathrm{~g})$ and $(\mathrm{h})$ ). This shifted hole peak in the gain spectrum could be see as an antihole around $\omega_{m}=0$. Thus, the signal delay could be explained in terms of this antihole. In summary, the behavior of the signal described above can be explained in terms of the interaction between the signal and the population oscillations generated by the pump. In some way, we could say that the pump intensity profile is imprinted onto the signal one through the population oscillations.

\subsection{Signal and pump delay coupling}

Now, let us analyze the interplay between the delay undergone by the signal beam and the one experienced by the pump beam, i.e., we study simultaneously the group velocity of both beams and the influence of the relative phase $\varphi$ in those velocities. We analyze under which conditions the coupling between the two beams makes that both propagate with the same group velocity, that is, the conditions that lead to a synchronization-like phenomenon. To this end, let us first analyze in more detail the relation between the analytical expression of the signal phase shift derived in equation (13) and the expression derived for the pump phase shift following the same approach:

$\phi_{p}(z=L) \simeq \frac{\alpha_{p} P_{p 0}}{\left(w_{c}^{2}+w_{m}^{2}\right) w_{c}\left(1+\beta_{s}\right)}\left[-w_{m}\left(1+\beta_{s}+\beta_{s} P_{s 0}\right)+\frac{P_{s m}}{P_{p m}}\left(\beta_{s} P_{p 0}-1\right) \sin \left[\varphi-\xi_{m}\right]\right]$

We look for a condition which allows both beams to travel with the same group velocity independently of the average pump or signal powers $\left(P_{p 0}\right.$ and $\left.P_{s 0}\right)$. To this 
end, we consider the case where the two beams are modulated in phase or in opposite phase, i.e., $\varphi=n \pi$ with $n=0,1$. Then, the signal and pump phase shifts remain:

$\phi_{s}(z=L) \simeq \frac{\alpha_{s} P_{s 0} w_{m}}{\left(w_{c}^{2}+w_{m}^{2}\right) w_{c}}\left[\left(\beta_{s} P_{p 0}-1\right)-\frac{P_{p m}}{P_{s m}}\left(1+\beta_{s}+\beta_{s} P_{s 0}\right)(-1)^{n}\right]$
$\phi_{p}(z=L) \simeq \frac{\alpha_{p} P_{p 0} w_{m}}{\left(w_{c}^{2}+w_{m}^{2}\right) w_{c}\left(1+\beta_{s}\right)}\left[\frac{P_{s m}}{P_{p m}}\left(\beta_{s} P_{p 0}-1\right)(-1)^{n}-\left(1+\beta_{s}+\beta_{s} P_{s 0}\right)\right]$

An inspection of equations (17)-(18) reveals that both beams suffer the same delay (in absolute value) when the ratio of the modulation depths is:

$$
\frac{P_{s m} / P_{s 0}}{P_{p m} / P_{p 0}}=\frac{\alpha_{s}\left(1+\beta_{s}\right)}{\alpha_{p}}
$$

which for our experimental system is close to 2 . Thus, $\phi_{s}=\phi_{p}$ at $\varphi=0$ and $\phi_{s}=-\phi_{p}$ at $\varphi=\pi$. To experimentally observe these predictions, we have measured simultaneously $F_{s}$ and $F_{p}$ as a function of the modulation frequency $f_{m}$ for different values of $\varphi$. We modulate the signal power $(1.8 \mathrm{~mW})$ with a modulation depth of $P_{s m} / P_{s 0} \simeq 0.65$ and the pump power $(2.2 \mathrm{~mW})$ with a modulation depth of $P_{p m} / P_{p 0} \simeq 0.3$ in such a way that the ratio between the modulation depths is close to the one given by equation (19). The experimental results (symbols) and the simulated ones (lines) are shown in figure $8 . F_{s}$ and $F_{p}$ are almost equal when both beams are modulated in phase, i.e., at $\varphi=0$ (see figure 8 (a)), in agreement with the theoretical prediction. In this situation, the coupling of both beams leads to similar signal and pump group velocities for all modulation frequencies. That means that both beams propagate in phase along the fiber. On the contrary, when we modulate both beams in phase opposition, i.e., at $\varphi=\pi, F_{s}$ and $F_{p}$ take the same value but with different sign, in agreement with the theoretical prediction, that is, while the signal suffers advancement the pump suffers a delay of the same value being the optimum modulation frequency around $100 \mathrm{~Hz}$ (see figure 8 (c)). More complex behaviors are observed at other values of the phase difference between both modulations, as shown in figure 8 (b) and (d). For example, at $\varphi=\pi / 2$, the pump beam suffers a transition from advancement to delay as the modulation frequency increases. Note that only the case of exactly equal velocities $(\varphi=0)$ is stable under propagation. However, for the other values of $\varphi$, within the undepleted approximation, the difference between the signal and pump phase shifts will depend linearly with the fiber length although this dependence is not relevant in our experiments due to the small values of $F_{s}$ or $F_{p}$ obtained.

We have also experimentally corroborated that, when both beams are modulated in phase $(\varphi=0)$ or in opposite-phase $(\varphi=\pi)$, they propagate with the same group velocity (in absolute value) independently of the average pump power. These results are shown in figure 9 where we plot the fractional advancement of the signal and pump as a function of the average pump power for these two relative phases $\varphi$ and for a modulation frequency of $f_{m}=50 \mathrm{~Hz}$. As in figure 8, we use again a signal power of $1.8 \mathrm{~mW}$ with a modulation depth of 0.65 , and a modulation depth for the pump power of 0.3 . We also plot the simulated fractional advancement of the signal (dashed line) and pump (solid line). As expected, the values of $F_{s}$ an $F_{p}$ are quite similar for each pump power when both beams are modulated in phase. Furthermore, when we modulate both beams in phase opposition, the fractional delay of the pump shows the same value than the fractional advancement of the signal and vice versa. As we 

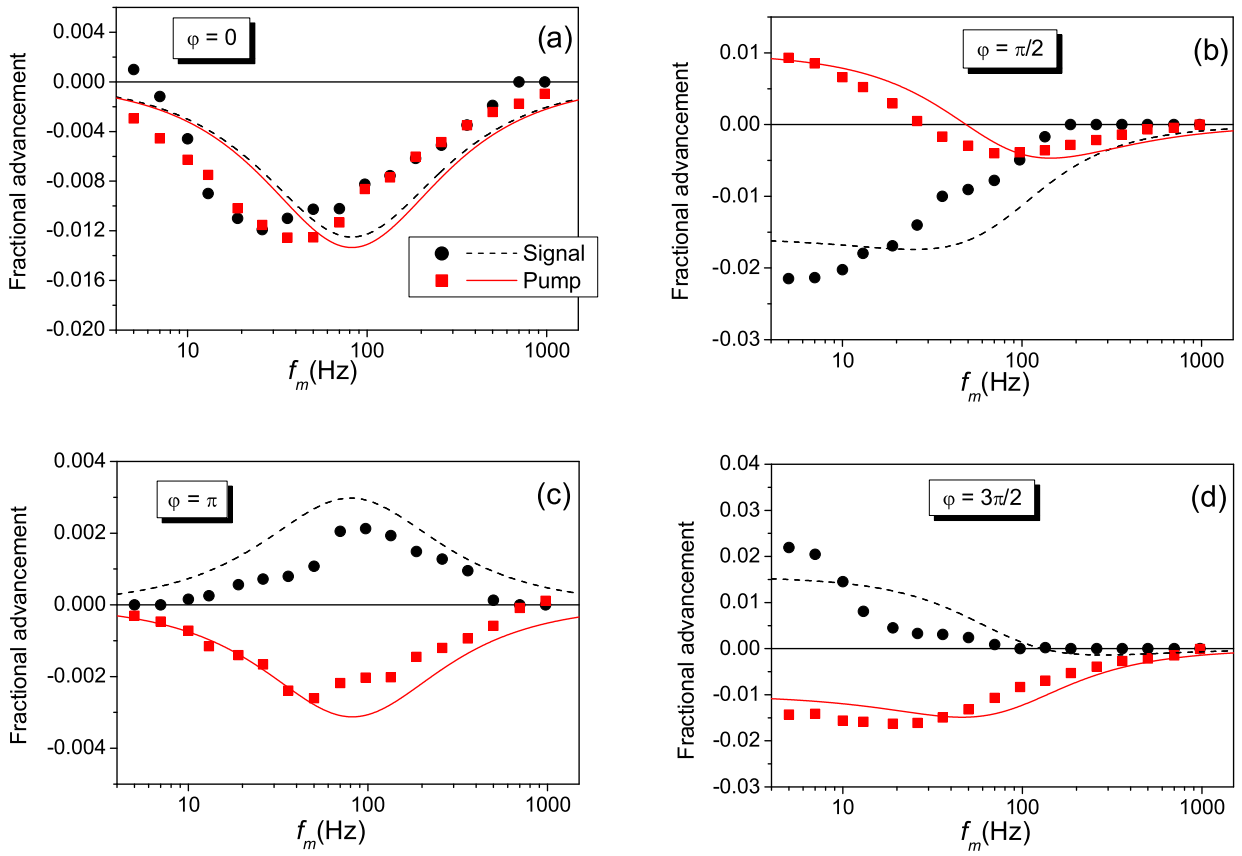

Figure 8. Fractional advancement of the signal (circles) and pump (squares) as a function of the modulation frequency for different relative phases between the signal and pump modulated beams $\varphi$. Simulated fractional advancement of the signal (dashed line) and pump (solid line). Signal power $=1.8 \mathrm{~mW}\left(P_{s 0}=3\right)$ with $P_{s m}=0.65 P_{s 0}$, and pump power $=2.2 \mathrm{~mW}\left(P_{p 0}=0.9\right)$ with $P_{p m}=0.3 P_{p 0}$.

have shown, the in-phase propagation of both beams is a general trend that does not depend on the modulation frequency or the average pump power, so it could be used for the propagation of pulses.

The group velocity synchronization-like phenomenon described in this work could be applied to a more interesting situation where we use two amplitude-modulated light beams, both with carrier frequencies within the optical communications $\mathrm{C}$ band (approximately from 1530 to $1565 \mathrm{~nm}$ ). In fact, as we mention in section 1, Stepanov et al [19] controlled the phase shift of a $1526 \mathrm{~nm}$ probe beam in an EDF by using a $1568 \mathrm{~nm}$ saturating beam. Following the same analysis used in this section, we could achieve the same group velocity for both beams when the ratio of the modulation depths satisfy the following condition:

$$
\frac{P_{s m}^{(1)} / P_{s 0}^{(1)}}{P_{s m}^{(2)} / P_{s 0}^{(2)}}=\frac{\alpha_{s}^{(1)}\left(1+\beta_{s}^{(1)}\right)}{\alpha_{s}^{(2)}\left(1+\beta_{s}^{(2)}\right)},
$$

where the superscripts (1) and (2) correspond to the different wavelengths. 

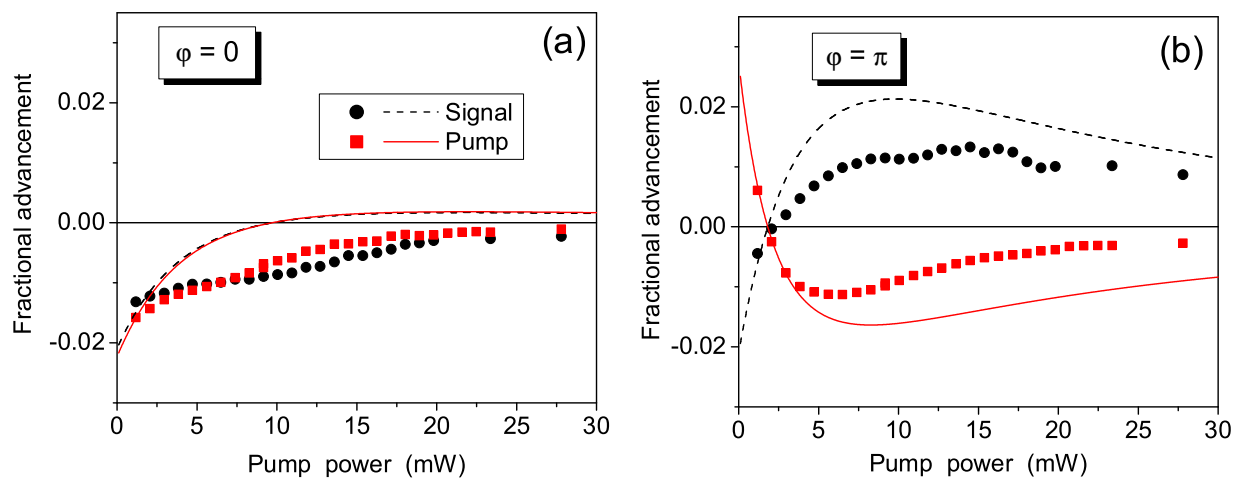

Figure 9. Fractional advancement of the signal (circles) and pump (squares) as a function of the average pump power for two different relative phases between the signal and pump modulated beams $\varphi$. Simulated fractional advancement of the signal (dashed line) and pump (solid line). Signal power $=1.8 \mathrm{~mW}\left(P_{s 0}=\right.$ 3) with $P_{s m}=0.65 P_{s 0}$, and $P_{p m}=0.3 P_{p 0} \cdot f_{m}=50 \mathrm{~Hz}$

\section{Conclusions}

In conclusion, we report the control of the slow and fast light propagation velocity of two amplitude-modulated light beams by means of CPO. These light beams correspond to the usual signal (at $1550 \mathrm{~nm}$ ) and pump (at $980 \mathrm{~nm}$ ) beams used in EDF amplifiers. To this end, we induce a simultaneous beat in both beams with the same frequency so that the relative phase between both beats allows us to control their group velocities. This condition could make difficult the application of the proposed mechanism for unpredictable signals, like a data stream. When the population oscillations are mainly governed by the modulated pump, an enhancement of the delay or advancement of the $1550 \mathrm{~nm}$ beam in one order of magnitude is observed. When the two modulated beams contribute similarly to the population oscillations, a synchronized propagation of both beams has been found. Thus, when both beams are modulated in phase, they propagate with the same group velocity along the fiber for each modulation frequency. This simultaneous propagation is obtained for all the average pump powers. Furthermore, when we modulate both beams in phase opposition, while signal suffers advancement, pump suffers delay, traveling both with the same absolute value of the group velocity. These results could be used in semiconductors which are good candidates for telecommunication applications due to their fast characteristic relaxation time scale (GHz). In fact, Antón et al [23] have shown that by modulating the injection current in a semiconductor optical amplifier to force the population oscillations they control and enhance the delay of the optical signal. These predictions have been experimentally proved in a very recent work [24]. 


\section{Acknowledgments}

This work has been supported by projects MEC-FIS2007-65382 and BSCH-UCMGR58/08: 910133-1030. We would like to thank F. Arrieta-Yáñez, M. A. Antón, F. Carreño, J. M. Ezquerro, and E. Cabrera-Granado for fruitful discussions and scientific support.

\section{References}

[1] Adamczyk O H, Cardakli M C , Cai. J-X, Hayee M I, Kim C and Willner A E 1999 IEEE Photon. Technol. Lett. 111057

[2] Gehring G M, Boyd R W, Gaeta A L, Gauthier D J, Willner A E 2008 J. Lightwave Technol. 263752

[3] Song K Y, Herráez M G, and Thèvenaz L 2005 Opt. Express 1382

[4] Okawachi Y, Bigelow M S, Sharping J E, Zhu Z, Schweinsberg A, Gauthier D J, Boyd R W and Gaeta A L 2005 Phys. Rev. Lett. 94153902

[5] Sharping J E, Okawachi Y and Gaeta A L 2005 Opt. Express 136092

[6] Ghosh S, Sharping J E, Ouzounov D G, Gaeta A L 2005 Phys. Rev. Lett. 94093902

[7] Bigelow M S, Lepeshkin N N and Boyd R W 2003 Phys. Rev. Lett. 90113903

[8] Hillman L W, Boyd R W, Kransinski J and Stroud Jr C R 1983 Opt. Commun. 45416

[9] Schweinsberg A, Lepeshkin N N, Bigelow M S, Boyd R W and Jarabo S 2006 Europhys. Lett. 73218

[10] Melle S, Calderón O G, Carreño F, Cabrera E, Antón M A and Jarabo S 2007 Opt. Commun. 27953

[11] Zhang Y, Qiu W, Ye J, Wang N, Wang J, Tian H and Yuan P 2008 Opt. Commm. 2812633

[12] Qiu W, Zhang Y, Ye J, Wang N, Wang J and Yuan P 2008 Appl. Optics 471781

[13] Gehring G M, Schweinsberg A, Barsi C, Kostinski N and Boyd R W 2006 Science 312895

[14] Shin H, Schweinsberg A, Gehring G M, Schwertz K, Chang H J, Boyd R W, Park Q-H and Gauthier D J 2007 Opt. Lett. 32906

[15] Shin H, Schweinsberg A and Boyd R W 2009 Opt. Comm. 2822085

[16] Melle S, Calderón O G, Caro C E, Cabrera-Granado E, Antón M A and Carreño F 2008 Opt. Lett. 33827

[17] Calderón O G, Melle S, Antón M A, Carreño F, Arrieta-Yáñez F and Cabrera-Granado E 2008 Phys. Rev. A $\mathbf{7 8} 053812$

[18] Arrieta-Yánẽz F, Melle S, Calderón O G, Antón M A and Carreño F 2009 Phys. Rev. A 80 011804(R)

[19] Stepanov S and Hernández E. H 2008 Opt. Lett. 332242

[20] Bigelow M S, Lepeshkin N N and Boyd R W 2003 Science 301200

[21] Boyd R W, Gauthier D J and Gaeta A L 2006 Opt. Photon. News 1718

[22] Wysocki P F, Wagener J L, Digonnet M J F and Shaw H J 1992 Proc. SPIE, Fiber Laser Sources and Amplifiers IV $\mathbf{1 7 8 9} 66$

[23] Antón M A, Carreño F, Calderón O G, Melle S, Arrieta-Yáñez F 2009 J. Phys. B: At. Mol. Opt. Phys. 42095403

[24] Berger P, Bourderionnet J, Valicourt G, Brenot R, Bretenaker F, Dolfi D, Alouini M 2010 Opt. Lett. 352457 